\title{
Lifestyle approaches for people with intellectual disabilities: A systematic multiple case analysis
}

\author{
Steenbergen H.A ${ }^{1,5}$, Van der Schans C.P. 1,2,5, Van Wijck R. ${ }^{4}$, De Jong J. ${ }^{3}$, Waninge A. \\ Hanze University of Applied Sciences Groningen, Groningen, the Netherlands \\ Department of Rehabilitation Medicine, University of Groningen, University Medical Center Groningen, Groningen, the Netherlands \\ . Center of Human Movement Sciences, University of Groningen, University Medical Center Groningen, Groningen, the Netherlands \\ 5. Department of Health Psychology, University of Groningen, University Medical Center Groningen, Groningen, the Netherlands
}

\section{Aim}

To analyze the intervention components, levels of influence, explicit use of theory, and conditions for sustainability of currently used lifestyle interventions within lifestyle approaches aiming at physical activity and nutrition in healthcare organizations supporting people with Intellectual Disabilities (ID)

\section{Conclusion}

Comprehensive, integrated, and theory-driven approaches at multiple levels should be promoted in healthcare organizations for people with ID.

\section{Background}

Healthcare organizations carry out a range of interventions to support and improve a healthy lifestyle.

However, it is difficult to implement and maintain active and healthy lifestyle into daily support.

It is unknown to what extent factors for implementation are taken into consideration by healthcare organizations.

\section{Methods}

Design: descriptive multiple case study

Research units: 9 healthcare organizations

Data collection: newly developed online inventory form (qualitative data).

Data procedure: analysis at four essential factors for implementation in practice:

- Multiple intervention components $\mathbf{s}^{1,2}$

- Multiple levels of influence $3,1,2,4$

- Explicit use of theory $5^{5,2,4}$

- Comprehensive written lifestyle policy ${ }^{3,6}$
Results

Nine healthcare organizations, 59 interventions

- Aims (improve); 31\% physical activity, $10 \%$ nutrition, $59 \%$ a combination of both.

- Aimed at; $49 \%$ educational component, $19 \%$ daily activities, $16 \%$ generic activities, $16 \%$ evaluation component

- Target groups; 38\% individuals with ID, 37\% professionals, $25 \%$ social levels

- Structurally embedded; $52 \%$

- Theory-driven: $17 \%$

- Lifestyle policy; $44 \%$ clear vision, $67 \%$ policy on lifestyle, $67 \%$ employees , $56 \%$ individual lifestyle-plans

- Funding; between $33 \%$ and $100 \%$ funded by own organizations,

Table 1. The lifestyle policy, the currently used components targeting the individual, professional, and social level, horizontal, within each healthcare organization, vertical. ( $i=$ individuals $\mathrm{ID}, \mathrm{p}=$ professional, $s=$ social). The gray colored cells represent the presence of a vision and policy on lifestyle, the presence of employees responsible for the theme lifestyle, and the presence of individual lifestyle plans, monitoring of adherence,, as well as the presence of the components per level within the healthcare organization (to read by row). The organization with the most components is listed at the top; we listed the organization with the fewest components at the bottom. The second and third row depict the number of interventions, combined

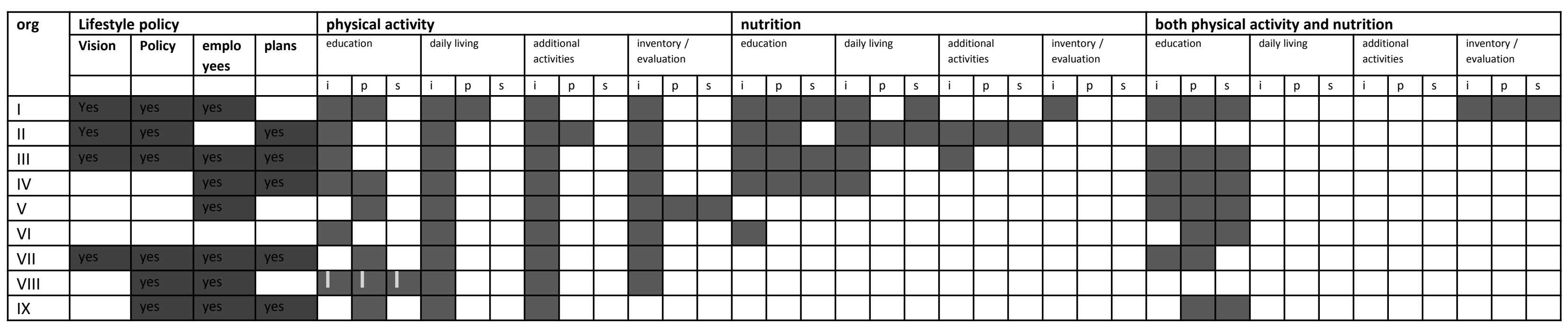

\section{References}

1.Sallis, J. F., Cervero, R. B., Ascher, W., Henderson, K. A., Kraft, M. K., \& Kerr, J. (2006). An ecological approach to creating active living communities. Annual Review of Public Health, 27, 297-322. doi:10.1146/annurev. publhealth.27.021405.102100. 2. Bartholomew L. K., Parcel G. S., Kok G., Gottlieb N. H. \& Fernaindez M. E. (2011) Planning Health Promotion Programs; An Intervention Framework. Americican Jounal of Public Health. September 1999, Vol. 89, No. 9. 4. . Naaldenberg, J., Kuijken, N., van Dooren, K., \& de Valk, , H. V. . S. L. (2013). Topics, methods and challenges in health promotion for people with intellectual disabilities: A structured review of literature. Research in Developmental Disabilities, 34(12), 4534-4545. 5. Gardner B, Whittington C, McAteer J,

Contact Details

Rianne Steenbergen h.a.steenbergen@pl.hanze.nl 